\title{
Maternal Knowledge of Tuberculosis and Bacillus Calmette Guerin Vaccination in Pediatric Health Services in Kinshasa
}

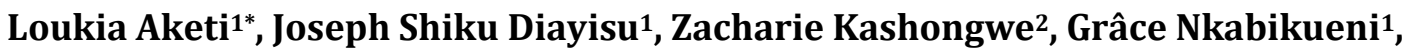 \\ Patrick Kayembe Kalambay ${ }^{3}$, Jean-Marie Kayembe ${ }^{2}$ \\ ${ }^{1}$ Cardio-Pulmonary and Infectious Diseases Unit, Department of Pediatrics, University Hospital of Kinshasa, \\ University of Kinshasa, Kinshasa, Democratic Republic of Congo \\ ${ }^{2}$ Pneumology Service, Department of Internal Medicine, University Hospital of Kinshasa, University of Kinshasa, \\ Kinshasa, Democratic Republic of Congo \\ ${ }^{3}$ Epidemiology and Biostatistics, Public Health School, University of Kinshasa, Kinshasa, Democratic Republic of Congo \\ Email: *akelou@yahoo.fr
}

How to cite this paper: Aketi, L., Diayisu, J.S., Kashongwe, Z., Nkabikueni, G., Kalambay, P.K. and Kayembe, J.-M. (2017) Maternal Knowledge of Tuberculosis and Bacillus Calmette Guerin Vaccination in Pediatric Health Services in Kinshasa. Journal of Tuberculosis Research, 5, 30-43. https://doi.org/10.4236/jtr.2017.51004

Received: January 5, 2017

Accepted: February 28, 2017

Published: March 3, 2017

Copyright ( 92017 by authors and Scientific Research Publishing Inc. This work is licensed under the Creative Commons Attribution International License (CC BY 4.0).

http://creativecommons.org/licenses/by/4.0/

\begin{abstract}
Introduction: To participate effectively in the fight against tuberculosis (TB), mothers need to have a good knowledge of TB and its prevention. The objective of this study was to evaluate the knowledge of mothers about $\mathrm{TB}$ and $\mathrm{Ba}$ cillus Calmette Guérin (BCG). Methods: This was a cross-sectional survey performed from September to December 2015 in 4 health care facilities of Kinshasa. It concerned mothers of children who received BCG vaccine. The frequencies and chi-square of Pearson were used to report results. Results: A total of 380 couples of the children and their mothers were recruited. The median age of children was 16 months (ranges: 6 days to 59 months); 224 $(58.9 \%)$ of them received BCG during the period recommended; $62(16.3 \%)$ experienced a side effect. There was a significant association $(p=0.00)$ between sides effects and the delayed vaccination. The average age of the mothers was $29.3 \pm 6.4$ years; 352 (92.6\%) had heard about TB; 28 (7.4\%) never heard about it; 168 (44.2\%) knew that TB is a contagious disease; only 111 (29.2\%) knew the mode of transmission; 87 (22.9\%) did not know any signs of $\mathrm{TB}$, and 54 (14.2\%) knew about prevention with the BCG vaccine. Factors significantly associated with the mothers' lack of knowledge were a low level of education $(p=0.01)$, young age $(p=0.02)$, and place of residence $(p=$ 0.04). Conclusion: There is an urgent need to improve the education of the population, particularly those who lives in poor conditions and who are uneducated.
\end{abstract}

\section{Keywords}

Maternal Knowledge, Tuberculosis, BCG, Children 


\section{Introduction}

Tuberculosis (TB) is one of the most severe infectious diseases in the world, particularly in Sub-Saharan African countries [1]. Its morbidity and mortality remain high, making it one of the three primary poverty-related infectious diseases [1] [2] [3]. The 2014 world objectives aimed for a $95 \%$ reduction in mortality and a $90 \%$ reduction in the incidence of TB compared with the rates of previous years and to bend the curve of incidence to eliminate TB as a public health problem before 2050 that means less than one case per one million inhabitants [4]. The World Health Organization (WHO) goal is that by 2020 no family should have to support the cost of TB [5]. The methods in the fight against this disease include prevention by vaccination with Bacillus Calmette Guérin (BCG) and the well-established curative treatment with antiTB drugs [6]. Although childhood $\mathrm{TB}$ is the reflection of the adult $\mathrm{TB}$ [7], there nevertheless persist some gaps in the management of TB in this younger population [8]. Because they are close to children, the involvement of parents, and especially of mothers, in the care of their children, occupies an important place in the fight against childhood TB. In Sub-Saharan countries, a substantial number of women are illiterate [9], which can constitute an obstacle in the adherence to antiTB guidelines and the achievement of global objectives.

Currently, in developing countries, the management of childhood TB is challenging [3]. The Democratic Republic of Congo (DRC), a high resourced setting, carries the 3rd largest burden of TB in Africa [10]. Therefore, it is necessary to evaluate mothers' knowledge of TB and TB prevention in an environment where TB occupies an important place in infantile morbidity and mortality. The objective of the study is to evaluate mothers' knowledge of TB and vaccination with BCG and to determine the factors associated with that knowledge.

\section{Methods}

This was a cross-sectional survey of mothers who sought consultation for their children aged 6 days to 5 years-old. The study was conducted from September to December 2015, four health care facilities that provided pediatric service. The facilities were chosen from the 4 health districts of the city of Kinshasa (Hospital Center of Kingasani, Pediatric Hospital of Kalembelembe, Maternity Hospital of Kintambo, and Hospital Center of Mont Amba). For that, we used a simple stratified sampling: the 4 health districts of Kinshasa city were considered as (first level) strats. From each strat we listed all health care facilities that provided a service of pediatrics and where the mean number of children's consultation was more than $30 \pm 10$ per day. We found an average 5 health care per district from what we selected randomly 1 health care facility. We had then 4 health care facilities to study. This procedure assured a consistent representatively to the statistical units. The sample size was calculated at 360 cases and was increased by $10 \%$ to account for losses for a total sample size of 396 cases. The value was calculated based on of the average number of pediatric consultations in those facilities. We recruited random sample by selecting each odd number in the registration of 
consultations. We included mothers of children aged 6 days to 5 years-old who received BCG vaccination, and who brought their children to the health care facility for consultation, and who signed the consent form for the study procedures. We excluded mothers who didn't remember about the BCG vaccination of their children. In consideration of the national guidelines, all newborn children have to receive the BCG before exit from the maternity ward [11]. The participants enrolled in the study were interviewed using a standardized questionnaire that was prepared in French and translated into the main local language "Lingala". All the investigators were fluent in both languages. Socio-demographic parameters of the mothers (age, parity, residence, education, socioeconomic level, occupation, religion) and of the children (age, sex) were recorded, and the knowledge of the mothers of TB and the BCG vaccine, the day of the BCG administration, the presence of a BCG scar, the site of vaccination, the BCG's side effects, the reason for the current consultation, and the presence of the vaccination card and the knowledge of its contents. Regarding the knowledge of $\mathrm{TB}$, the mothers were asked 5 questions: if they had heard about TB, and to say how they heard about it and what is it, if the disease is contagious; what the mode of transmission is; if they knew the symptoms of $\mathrm{TB}$, and if yes to list them; and if TB is preventable, by vaccination or others measures, and to give the name of the vaccine.

The National Ministry of Health provides a vaccination card for all newborn children at first contact with the vaccination service. It contains the identity and the sex of the child, the weight at birth, the list of the vaccines to receive up to 9 months of age, including the planned dates and the administration dates of the vaccines, a growth curve with 3 different zones (normal zone in green, risk zone in yellow and danger zone in red), and a section of observations including different anthropometric parameters taken during preschool consultations.

The education level was characterized in 3 groups: the low education level for mothers who had not gone to school or who had not finished the elementary school studies; medium education level for those who had completed at least 3 years of secondary school or those who had obtained a high school diploma, and high education level for those who had completed at least 3 years of university education or institute. Additionally, the socioeconomic level was subdivided into 3 groups according to the Multidimensional Poverty Index (MPI) [12], low socioeconomic level, medium socioeconomic level and high socioeconomic level, on the basis of the possession of some household electrical assets such as a radio, television, or car, the number of rooms in the house, and access to potable water and the electricity at home. Occupations were grouped into 3 categories, first, workers (in the public or private sectors, liberal professions, or commercial professional activities), students (in school or professional study), and unemployed (housework) or those without activity. The parity was also divided into 3 groups, primiparity for the mothers having one child, the low multiparity for the mothers having 2 to 5 children, and the high multiparity concerned the mothers having more than 5 children. The residences was arbitrarily distributed into 4 groups on the basis of the level of development of the district and the presence of 
good asphalt roads, enclosed houses, and people possessing a car. We had 4 district level, going from 1 to 4 , the first one being the most developed. Level 5 included people living outside the city of Kinshasa and for whom it was difficult to define the level of the district.

Data were analyzed with SPSS 23.0 software. The measures of central tendency were determined for the variables of interest. The frequencies are presented in percentages and in absolute numbers. The chi-square of the Pearson correlation coefficient allowed the determination factors associated with the lack of knowledge in the mothers.

The authorization of the Ethics Committee of the Public Health School of Kinshasa was obtained before the start of the study; the informed consent of the mothers was also obtained before the start of this study. The questionnaires were anonymous and used only by the research team, and the recommendations of the Declaration of Helsinki were respected.

\section{Results}

A total of 396 mothers were recruited, but 16 (4.0\%) were excluded because they didn't remember about the BCG vaccination of their children. Finally, 380 pairs of children and their mothers were retained for the present study, and they came from the 4 health hospitals selected in the 4 health districts of Kinshasa.

\subsection{Socio-Demographic Data of Children and Mothers}

The median age of the children was 16 months (range: 6 days to 59 months). Male sex was prevalent at 213 (56.0\%), and 167 (44.0\%) children were female, so the sex ratio was 1.2. The average age of the mothers was $29.3 \pm 6.4$ years old, and the range was 15 to 48 years. Among them, 10 (20.6\%) refused to declare their age and $2(0.5 \%)$ mothers did not know their age. More than half of the mothers, 216 (56.8\%), had low multiparity, the majority of them, 257 (67.6\%), worshiped in liberal Christian churches, $174(45.8 \%)$ had a medium level of education, almost half, 195 (51.3\%), were workers, and 162 (42.6\%) were unemployed. The distribution of mothers in the different district levels was equivalent. Table 1 gives details of the socio-demographic characteristics of the mothers (Table 1).

\subsection{Clinical Data of the Children}

Fever was the reason for consultation in 197 (51.8\%) children, cough in 49 cases (12.9\%), digestive symptoms (diarrhea and/or vomiting) in 37 cases $(9.7 \%)$, symptoms related to nutritional problems (anorexia and/or loss of weight) in 28 cases (7.4\%), symptoms related to hematological problems (paleness and/or bleeding) in 22 cases (5.8\%), a routine examination and/or a control in 17 cases (4.5\%), a surgical cause in 15 cases (3.9\%) most of which were the demand of circumcision (13 cases or $3.4 \%$ ), a hernia (umbilical and inguino-scrotal) for 2 cases $(0.5 \%)$, a skin rash in 11 cases $(2.9 \%)$, and a neurological symptoms (headaches or persistent crying or cranial palsy) in 4 cases (1.1\%). 
Table 1. Distribution of the mothers according to the socio-demographic characteristics and the children according to the clinical data.

\begin{tabular}{|c|c|c|}
\hline Mothers characteristics & Frequency & $\%$ \\
\hline \multicolumn{3}{|l|}{ Age groups } \\
\hline $15-24$ years & 95 & 25.0 \\
\hline 25 - 35 years & 184 & 48.4 \\
\hline 35 - 44 years & 81 & 21.3 \\
\hline $45-54$ years & 8 & 2.1 \\
\hline Unknown & 12 & 3.1 \\
\hline \multicolumn{3}{|l|}{ Education $^{*}$} \\
\hline Low & 91 & 23.9 \\
\hline Medium & 174 & 45.8 \\
\hline High & 115 & 30.3 \\
\hline \multicolumn{3}{|l|}{ Occupation } \\
\hline Worker & 195 & 51.3 \\
\hline Student & 23 & 45.8 \\
\hline Unemployed & 162 & 30.3 \\
\hline \multicolumn{3}{|l|}{ Religion } \\
\hline Catholic & 63 & 16.6 \\
\hline Protestant & 42 & 11.1 \\
\hline Liberal Christian Churches & 257 & 67.6 \\
\hline Others $^{*}$ (Muslim, Kimbaguist...) Parity ${ }^{\epsilon}$ & 18 & 4.7 \\
\hline Primiparity & 112 & 29.5 \\
\hline Low multiparity & 216 & 56.8 \\
\hline High multiparity & 52 & 13.7 \\
\hline \multicolumn{3}{|l|}{ Residence $^{\mathfrak{E}}$} \\
\hline Level 1 & 77 & 20.3 \\
\hline Level 2 & 99 & 26.1 \\
\hline Level 3 & 107 & 28.2 \\
\hline Level 4 & 93 & 24.5 \\
\hline Level 5 & 4 & 1.1 \\
\hline \multicolumn{3}{|l|}{ Motive of children's consultation } \\
\hline Fever & 197 & 51.8 \\
\hline Cough & 49 & 12.9 \\
\hline Digestive symptoms (diarrhea and/or vomiting) & 37 & 9.7 \\
\hline Nutritional problems (anorexia and/or loss of weight) & 28 & 7.4 \\
\hline Hematological problems (paleness and/or bleeding) & 22 & 5.8 \\
\hline Routine examination and/or a control & 17 & 4.5 \\
\hline Surgical cause & 15 & 3.9 \\
\hline Skin rash & 11 & 2.9 \\
\hline Neurological symptoms (headaches or persistent crying or cranial palsy) & 4 & 1.1 \\
\hline
\end{tabular}

Legend: *low level: no study or did not complete elementary school studies; medium level: at least 3 years of secondary school or a high school diploma; high level: at least 3 years of university education or institute. ${ }^{\sharp}$ Others: Kimbanguist, Muslim, "Church of Black in Congo", atheist; ${ }^{6}$ Primiparity: mother having 1 child, low multiparity: mother having 2 to 5 children, high multiparity: mother having more than 5 children; ${ }^{\text {E }}$ Level 1: at least $3 / 4$ of the roads have asphalt, most people have a car, and the majority of houses are enclosed; level 2: at least $1 / 2$ of the roads have asphalt, some people have a car, and at least half of the houses are enclosed; level 3: at least $1 / 4$ of the roads have asphalt, few people have a car, and few houses are enclosed; level 4: less than 1/4 (or none) of the roads have asphalt, the majority of people do not have a car, and almost none of the houses are enclosed; level 5: all people who live outside Kinshasa city. 
In total, 224 (58.9\%) children had received the BCG vaccination during the period recommended by the National Program of Vaccination (NPV); this period is during the 14 days following birth, before baby is brought into the community. However 147 (38.7\%) received it between 15 days and 3 months, and 1 $(0.3 \%)$ child had received it after the age of 3 months. Her mother justified the delay as being due to her travel to a nearby country for commercial activities. For 8 (2.1\%) children, the mothers did not remember the day of BCG administration. In 269 (70.8\%) cases, there was a scar from the BCG, and in 111 (29.2\%), the scar was absent. The national TB program in DRC (NTP) recommends the administration of BCG in the left forearm, with the right being reserved for the Mantoux test. Showing that this convention was observed, the BCG was administered in the left forearm in 377 (99.2\%) cases and in the right forearm in 3 $(0.8 \%)$ cases. The mothers of these 3 children were not aware of the reason for the choice of this unusual site. In total, 318 (83.7\%) mothers declared to have observed no side effects, 58 (15.2\%) mothers had observed fever, and 4 (1.1\%) mothers had observed a subcutaneous abscess.

We compared the presence or absence of side effects, the presence of a BCG scar, and the day of administration of BCG. These results showed a significant association $(p=0.004)$ between the sides effects and the day of vaccination, there was no association $(p=0.690)$ with the presence of the BCG scar (Table 2).

\subsection{Maternal Knowledge of TB and BCG Vaccination}

In total, 352 (92.6\%) mothers reported they had heard about TB, and 28 (7.4\%) had never heard about it, 168 (44.2\%) knew that TB is a contagious disease, and only 111 (29.2\%) mothers knew its mode of transmission. Concerning the symptoms/signs of TB, 227 (59.7\%) mothers reported at least one of the following signs: cough, fever, or loss of weight; 15 (3.9\%) mothers reported at least the anorexia, and/or the asthenia, and/or the night perspiration; $1(0.3 \%)$ mother reported adenopathy, but 87 (22.9\%) mothers did not know any signs of TB. Regarding TB prevention, $256(67.4 \%)$ mothers reported that TB can be prevented by immunization, 124 (32.6\%) mothers did not know this mode of prevention, and 54 (14.2\%) mothers cited BCG vaccination. The details about maternal knowledge are presented in Table 3.

Table 2. Association between side effects and the presence of a BCG scar and the day of vaccination.

\begin{tabular}{|c|c|c|c|c|c|}
\hline \multirow{2}{*}{ Parameters } & \multicolumn{4}{|c|}{ Age at BCG vaccination } & \multirow{2}{*}{$p$ value } \\
\hline & $0-14 \mathrm{~d}^{*}$ & $15 d^{*}-3$ months & $>3$ months & Do not know & \\
\hline Side effects & & & & & 0.004 \\
\hline No $(n=318)$ & $204(63.9 \%)$ & $106(33.2 \%)$ & $0(0.0 \%)$ & $8(0.25 \%)$ & \\
\hline Yes $(n=62)$ BCG Scar & $20(32.8 \%)$ & $41(67.2 \%)$ & $1(0.3 \%)$ & $0(0.0 \%)$ & 0.690 \\
\hline Yes $(n=269)$ & $155(57.6 \%)$ & $108(40.1 \%)$ & $1(0.4 \%)$ & $5(1.9 \%)$ & \\
\hline No $(n=111)$ & $69(62.2 \%)$ & $39(35.1 \%)$ & $0(0.0 \%)$ & $3(2.7 \%)$ & \\
\hline
\end{tabular}

Legend: ${ }^{\star} \mathrm{d}=$ days. 
Table 3. Distribution of mothers according to their knowledge of TB.

\begin{tabular}{|c|c|c|}
\hline \multirow{2}{*}{ Questions and answers } & \multicolumn{2}{|c|}{ Frequency } \\
\hline & $\mathrm{n}$ & $\%$ \\
\hline \multicolumn{3}{|l|}{ Did you heard about TB? } \\
\hline -Yes & 352 & 92.6 \\
\hline -No & 28 & 7.4 \\
\hline \multicolumn{3}{|l|}{ How did you heard about it? } \\
\hline -Hospital (maternity) & 243 & 63.9 \\
\hline -Friends (and/or neighbouring) & 26 & 6.8 \\
\hline -Family & 23 & 6.0 \\
\hline -Church & 5 & 1.3 \\
\hline -Media (television or radio) & 17 & 4.5 \\
\hline -Notice board & 18 & 4.7 \\
\hline -School & 9 & 2.4 \\
\hline -Children learned about it & 3 & 0.8 \\
\hline -Do not know & 36 & 9.5 \\
\hline \multicolumn{3}{|l|}{$\mathrm{Tb}$ : is it contagious? } \\
\hline -Yes & 168 & 44.2 \\
\hline -No & 212 & 55.8 \\
\hline \multicolumn{3}{|l|}{ What is its mode of transmission? } \\
\hline -Airway & 39 & 10.3 \\
\hline -Sputum & 34 & 8.9 \\
\hline -Drinking or eating with same crockery & 21 & 5.5 \\
\hline -Partner or friend with TB & 17 & 4.5 \\
\hline -Touching & 35 & 9.2 \\
\hline -Sleeping together & 38 & 10.0 \\
\hline -Sorcerer & 12 & 3.1 \\
\hline -Water & 53 & 13.9 \\
\hline -Punish of God & 27 & 7.1 \\
\hline -Conflict with other persons & 6 & 1.6 \\
\hline -Consequence of multiple sexual partners & 11 & 2.9 \\
\hline -Others* & 9 & 2.4 \\
\hline -Do not know & 78 & 20.5 \\
\hline \multicolumn{3}{|l|}{ Do you know signs of TB? } \\
\hline -Yes & 293 & 77.1 \\
\hline -No & 87 & 22.9 \\
\hline \multicolumn{3}{|l|}{ Can you list them? } \\
\hline -Cough, and/or fever, and/or loss of weight & 227 & 59.7 \\
\hline -Anorexia, and/or asthenia, and/or night perspiration & 15 & 3.9 \\
\hline -Others (adenopathy, respiratory distress) & 9 & 2.4 \\
\hline -Do not know & 129 & 33.9 \\
\hline
\end{tabular}




\section{Continued}

TB is it preventable by vaccination?

-Yes 256

-Do not know

124

Which vaccine?

$-B C G$

$54 \quad 14.2$

-Other vaccine (anti-poliomyelitis or diphtheria or pneumococcal)

-Do not know

TB: is it preventable by other procedure? If yes, which procedure?

-Yes

$302 \quad 79.5$

• Eat well

- Pry

- Clean the hands

- Do not cough

- Go to the hospital

- Take drugs

- Stop sexual activity

- Stop drink alcohol

- Do not breathe the same air than the person infected by TB

- Stop quarrel with neighbor

- Do not eat meet

- Give breastfeeding

-No

Do you bring the vaccination card to the consultation

-Yes

$\begin{array}{ll}50 & 13.2 \\ & \end{array}$

-No

Why not?

$\begin{array}{lcc}\text {-Not necessary } & 148 & 38.9 \\ \text {-Forgot } & 25 & 6.6 \\ \text {-Lost } & 38 & 10.0 \\ \text {-Never ask about that } & 115 & 30.3 \\ \text {-No response } & 54 & 14.2\end{array}$

Legend: ${ }^{*}$ Others: no-understandable causes.

In total, 372 (97.9\%) mothers had declared possessing a vaccination card for the children, 50 (13.2\%) brought this card to the consultation, and 330 (86.8\%) did not bring it. Only 7 (1.8\%) mothers knew the contents of this card, 281 (73.9\%) mothers had cited at least one content item of the card, and 92 (24.2\%) mothers did not know any of the contents.

Factors significantly associated with the mothers' lack of knowledge were low level of education $(p=0.01)$, age less than 24 years-old $(p=0.02)$, and residence $(p=0.04)$ (Table 4). 
Table 4. Factors associated with the mothers' lack of knowledge of TB.

\begin{tabular}{|c|c|c|c|c|}
\hline \multirow{3}{*}{ Parameters } & \multicolumn{3}{|c|}{ Mothers' knowledge of TB } & \multirow{3}{*}{$p$ value } \\
\hline & Yes $(n=256)$ & No $(\mathrm{n}=124)$ & Total $(\mathrm{n}=380)$ & \\
\hline & $\mathrm{n}(\%)$ & $\mathrm{n}(\%)$ & $\mathrm{n}(\%)$ & \\
\hline Age groups & & & & 0.02 \\
\hline $15-24$ years & $49(19.1)$ & $46(37.1)$ & $95(25.0)$ & \\
\hline $25-34$ years & $131(51.2)$ & $53(42.7)$ & $184(48.0)$ & \\
\hline 35 - 44 years & $63(24.6)$ & $18(14.5)$ & $81(21.3)$ & \\
\hline 45 - 54 years & $6(2.3)$ & $2(1.6)$ & $8(2.1)$ & \\
\hline Unknown & $7(2.8)$ & $5(4.0)$ & $12(3.1)$ & \\
\hline Education & & & & 0.01 \\
\hline Low level & 48 (18.8) & $43(34.7)$ & $91(23.9)$ & \\
\hline Medium level & $120(46.9)$ & $54(43.5)$ & $174(45.8)$ & \\
\hline High level & $88(34.4)$ & $27(21.8)$ & $115(30.3)$ & \\
\hline Occupation & & & & 0.78 \\
\hline Worker & $134(52.3)$ & $61(49.2)$ & $195(51.3)$ & \\
\hline Student & $16(6.3)$ & $7(5.6)$ & $23(6.1)$ & \\
\hline Unemployed & $106(41.4)$ & $56(45.2)$ & $162(42.6)$ & \\
\hline Residence & & & & 0.04 \\
\hline Level 1 & $51(19.9)$ & $26(21.0)$ & $77(20.3)$ & \\
\hline Level 2 & $78(30.5)$ & $21(16.9)$ & $99(26.1)$ & \\
\hline Level 3 & $59(23.0)$ & $48(38.7)$ & $107(28.2)$ & \\
\hline Level 4 & $64(25.0)$ & $29(23.4)$ & $93(24.5)$ & \\
\hline Level 5 & $4(1.6)$ & $0(0.0)$ & $4(1.1)$ & \\
\hline Religion & & & & 0.10 \\
\hline Catholic & $46(18.0)$ & $17(13.7)$ & $63(16.6)$ & \\
\hline Protestant & $33(12.9)$ & $9(7.3)$ & $42(11.1)$ & \\
\hline Liberal Christian Churches & $168(65.6)$ & $89(71.8)$ & $257(67.6)$ & \\
\hline Others $^{\star}$ (Kimbaguist, Muslim ...) & $9(3.5)$ & $9(7.3)$ & $18(4.7)$ & \\
\hline Parity & & & & 0.17 \\
\hline Primiparity & $68(26.6)$ & $44(35.5)$ & $112(29.5)$ & \\
\hline Low multiparity & $150(58.6)$ & $66(53.2)$ & $216(56.8)$ & \\
\hline High multiparity & $38(14.8)$ & $14(11.3)$ & $52(13.7)$ & \\
\hline
\end{tabular}

Legend: ${ }^{\star}$ low level: no education or did not complete elementary school studies, medium level: at least 3 years of secondary school or a high school diploma, high level: at least 3 years of university education or institute. ${ }^{*}$ Others: Kimbanguist, Muslim, Church of Black in Congo, atheist; ${ }^{6}$ Primiparity: mother having 1 child, low multiparity: mother having 2 to 5 children, high multiparity: mother having more than 5 children; $£$ Level 1 : at least $3 / 4$ of the roads have asphalt, most people have a car, and the majority of houses are enclosed; level 2: at least $1 / 2$ of the roads have asphalt, some people have a car, and at least half of the houses are enclosed; level 3: at least $1 / 4$ of the roads have asphalt, few people have a car, and few houses are enclosed; level 4: less than 1/4 (or none) of the roads have asphalt, the majority of people do not have a car, and almost none houses are enclosed; level 5: all people who live outside Kinshasa city. 


\section{Discussion}

The fight against TB in the world reached a decisive turning point with the "End TB" strategy [4]. Although efforts have been made to achieve zero mortality, childhood TB remains a challenge [13]. The WHO then altered its strategies [14] for the prevention and the control of the disease, and it requested the implementation of several modes of attack. The prevention of childhood TB means the precaution to avoid TB in children that includes the BCG vaccination, a contact screening and case-finding when there is contact with a TB patient, a primary prophylaxis with Isoniazid preventive therapy for infants and children living with Human Immunodeficiency virus who are considered unlikely to have $\mathrm{TB}$ and with no known exposure to $\mathrm{TB}$ [5], and precautions for a multidrug resistant TB mother to ovoid contagion of her children. Among this mode, the involvement of mothers occupies an important and essential place in better controlling of the childhood TB because mothers are close to their children and participate in much of the care of their children. This involvement will improve when mothers know about and have a good understanding of the disease. The purpose of this study was to estimate the level of TB knowledge in mothers in a country where TB is prevalent and where the management of childhood TB is a challenge [15].

The majority of the consultations in tropical countries concern infectious diseases. In this study, more than half of the children $(64.7 \%)$ had consulted for fever or cough, especially for respiratory infections; therefore, most of the children had symptoms related to infectious diseases. These symptoms are also symptoms of TB. Although mothers receive education on preventable diseases such as TB in each preschool consultation (CPS), the mothers did not know about TB, its modality of transmission or its prevention.

The BCG reduces the morbidity and the severe forms of $\mathrm{TB}$ in children [16]-[21]. This vaccine is obligatory in the DRC and is given at the maternity hospital with in the first 14 days after birth, with the goal of vaccinating all newborn. However, we noticed that this recommendation is not strictly followed, as there were cases excluded from the study because of the absence of BCG, and there were children who received it after 30 days for different reasons. This vaccine is well tolerated, except for some side effects, such as fever or subcutaneous abscesses, which were found in $18.7 \%$ of the cases in the present study. These side effects were correlated with the time of vaccination, especially when the vaccination was given after 14 days of age. The immaturity of the immune system in young newborn children, can explain the limitation of the local inflammatory reaction [22]. This result has not been found in other studies. In the United States in 1996, an observation made of people who had received the BCG noted a higher incidence of side effects in children younger than 1 year-old (387 cases), compared with those aged 1 to 20 years (25 cases), especially for local subcutaneous abscesses and regional adenopathy [23].

Although the BCG is widely administered in our country, only $14.2 \%$ of mothers knew about BCG, while $67.4 \%$ of the mothers knew that TB could be 
prevented by immunization. This observation showed the insufficiency of the mothers' knowledge of the first preventive measures given to their child. Similarly, 36.3\% of the mothers did not know the mode of $\mathrm{TB}$ transmission, and $22.9 \%$ of mothers did not know any signs of TB. This observation is worrying because the routine vaccination and informational campaigns have been conducted for several years in the DRC, and the mothers participates in the awareness messages during the CPS before and after the vaccination of their children. It is possible that more must be done to improve the mothers' knowledge, such as using television messages or home campaigns.

We observed that $92.6 \%$ of mothers declared knowledge of TB, in reality, only $44.2 \%$ knew that it is contagious and, $29.2 \%$ knew its mode of transmission; $22.9 \%$ did not know any signs of TB. This low level of knowledge was also reported in other studies. In a Saudi Arabian survey given to residents of Makkah and Jeddah in 2016, Ssedhar Al Otaibi et al. observed that a high numbers of participants did not know about TB; for example, only $15.2 \%$ of participants answered that TB can be prevented by vaccination [24]. The report of another 2016 study carried out in southern France showed that 48\% of parents knew about TB, but only $19 \%$ of them reported that BCG can protect against TB, and $73 \%$ agreed that vaccination protects against TB [25].

This survey also reported that poverty was significantly associated with the lack of TB knowledge because young age $(\mathrm{p}=0.02)$, low level of education $(\mathrm{p}=$ $0.01)$ and poor area of residence $(\mathrm{p}=0.04)$ were significantly associated with lack of knowledge about TB. In France in 2012, Bouhamam et al. demonstrated that socioeconomic factors related to poverty were significantly associated with lack of knowledge of immunizations. Indeed children who did not attend school $(\mathrm{OR}=2.5)$, who had parents who were not fluent in French $(\mathrm{OR}=5.7)$, who lived in caravans or were squatting $(\mathrm{OR}=11.5)$, or who were recipients of national medical assistance for foreigners $(\mathrm{OR}=12.8)$ had significant delays with some vaccines, but children who were recipients of the national medical assistance for citizens were better vaccinated against tuberculosis and hepatitis B [26]. A study in Uganda carried out in 2016 that aimed to survey mothers' knowledge and attitudes towards childhood immunizations showed that maternal knowledge correlated with complete childhood vaccination [27], and a 2006 study in Nigeria reported that mother's knowledge of immunization $(p=0.006)$ and vaccination at a privately funded health facility $(p<0.001)$ were significantly correlated with the rate of full immunization [28]. Therefore, there is a need to increase awareness through public education in the target groups of the population who live in poor conditions. One possibility is that health workers who prove their efficacy in remote settings can add TB awareness activities to their routine [29].

The mother's level of education has an important place in the comprehension and application of the recommendations proposed. Miyahara et al. [30] found that vaccination by day 7 was associated with living in urban areas (west rural: adjusted OR $(\mathrm{AOR})=6.13,95 \% \mathrm{CI}: 3.20-11.75$, east rural: $\mathrm{AOR}=6.72,95 \% \mathrm{CI}$ : 
3.66 - 12.33) and with maternal education (senior-educations ( $\mathrm{AOR}=2.43$, 95\%CI: 1.17 - 5.06). Schoeps et al. in Burkina Faso reported that the mother's education positively influenced timely adherence to the vaccination schedule $(\mathrm{OR}=1.42,95 \% \mathrm{CI} 1.06-1.89)$, but there was no effect of the age of the mother [31]. Globally, most authors agree that the socioeconomic and educational levels have an influence on the lack of knowledge of TB and vaccination. Improving public education on TB and its prevention can contribute to better control of the disease, similar to other diseases, such as malaria [32], where the intervention of trained community health workers has had a good impact on its control.

The result of this study showed that the education of people on TB is in sufficient. The program to fight against $\mathrm{TB}$ has to improve the method of educating people about preventable diseases, such as TB, which can help to reduce the disease's burden. However, we did not select mothers with children in contact with TB or who had suffered from $\mathrm{TB}$, and we did not explore other factors, such as the distance from the house to the health care facility.

\section{Conclusion}

This study revealed that $28(7.4 \%)$ mothers had never heard about TB; 168 (44.2\%) knew that it is a contagious disease; only 111 (29.2\%) knew its mode of transmission; 87 (22.9\%) did not know any signs of $\mathrm{TB}$, and 54 (14.2\%) knew about TB prevention with BCG vaccine. In bivariate analysis, low level of education $(p=0.01)$, young age $(p=0.02)$, and the place of residence $(p=0.04)$ were significantly associated with the mothers' lack of knowledge of TB. The WHO now advocates the "End TB" strategy, which aims to eliminate the disease before 2035. Therefore, it would be important to engage the actors in the fight, especially mothers, who are the primary caregivers and closest people to the children. There is an urgent need to improve the education of the population, particularly of those who live in poor conditions and who have uneducated backgrounds.

\section{Acknowledgements}

The authors thank the team of the Pneumology Service of the University Clinic of Kinshasa, doctors Bolie Grace, Bemba Joelle, Bundutidi Gloria and Matula Irène, for their valuable contributions to the collecting of data for this study.

\section{References}

[1] Bhutta, Z.A., Sommerfeld, J., Lassi, Z.S., Salam, R.A. and Das, J.K. (2014) Global Burden, Distribution, and Interventions for Infectious Diseases of Poverty. Infectious Diseases of Poverty, 3, 21. https://doi.org/10.1186/2049-9957-3-21

[2] World Health Organization (2012) Global Report for Research on Infectious Diseases of Poverty. Geneva. http://whqlibdoc.who.int/publications/2012/9789241564489_eng.pdf

[3] Seddon, J.A. and Shingadia, D. (2014) Epidemiology and Disease Burden of Tuberculosis in Children: A Global Perspective. Infection and Drug Resistance 7, 153-165.

[4] World Health Organization (2015) The End TB Strategy. Geneva. 
[5] World Health Organization (2014) Guidance for National Tuberculosis Programs on the Management of Tuberculosis in Children. 2nd Edition, Geneva.

[6] World Health Organization (2006) Guidance for National Tuberculosis Programs on the Management of Tuberculosis in Children. Geneva.

[7] Bloch, A.R. and Snider, D.E.J. (1986) How Much Tuberculosis in Children Must We Accept? American Journal of Public Health, 76, 14-15. https://doi.org/10.2105/AJPH.76.1.14

[8] Aketi, L., Shiku, D.J., Kashongwe, Z., Lay, G., Kibadi, K. and Kayembe, K.P. (2016) Trends in Tuberculosis Epidemiology among Children in the Democratic Republic of Congo. Journal of Tuberculosis Research, 4, 235-248.

[9] UNESCO (2015) Alphabétisme des adultes et des jeunes. Bulletin d'information de l'Institut de Statistique de l'UNESCO.

[10] World Health Organization (2016) Global Tuberculosis Report. Geneva.

[11] République Démocratique du Congo (2014) Ministère de la santé-Programme élargi de vaccination. Plan Pluri Annuel Complet du PEV de la République Démocratique du Congo, 2015-2019.

[12] Union Nations Development Program (2015) Human Development Report 2015: Work for Human Development.

[13] Elhassan, M.M., Elmekki, M.A., Osman, A.L. and Hamid, M.E. (2015) Challenges in Diagnosing Tuberculosis in Children: A Comparative Study from Sudan. Inter- national Journal of Infectious Diseases, 43, 25-29.

[14] World Health Organization (2014) Guidance for National Tuberculosis Programs on the Management of Tuberculosis in Children. 2nd Edition, Geneva.

[15] Aketi, L., Kashongwe, Z., Kinsiona, C., Fueza, S.B., Kokolomami, J., Bolie, G., et al. (2016) Childhood Tuberculosis in a Sub-Saharan Tertiary Facility: Epidemiology and Factors Associated with Treatment Outcome. PLoS ONE, 11, e0153914. https://doi.org/10.1371/journal.pone.0153914

[16] Romanus, V. (1987) Tuberculosis in Bacillus Calmette-Guerin-Immunized and Unimmunized Children in Sweden: A Ten-Year Evaluation Following the Cessation of General Bacillus Calmette-Guerin Immunization of the Newborn in 1975. The Pediatric Infectious Disease Journal, 6, 272-280. https://doi.org/10.1097/00006454-198703000-00014

[17] Padungchan, S., Konjanart, S., Kasiratta, S., Daramas, S. and Ten Dam, H.G. (1986) The Effectiveness of BCG Vaccination of the Newborn against Childhood Tuberculosis in Bangkok. Bulletin of the World Health Organization, 64, 247-258.

[18] Tidjani, O., Amedome, A. and Ten Dam, H.G. (1986) The Protective Effect of BCG Vaccination of the Newborn against Childhood Tuberculosis in an African Community. Tubercle, 67, 269-281. https://doi.org/10.1016/0041-3879(86)90016-4

[19] Young, T.K. and Hershfield, E.S. (1986) A Case-Control Study to Evaluate the Effectiveness of Mass Neonatal BCG Vaccination among Canadian Indians. American Journal of Public Health, 76, 783-786. https://doi.org/10.2105/AJPH.76.7.783

[20] Shapiro, C., Cook, N., Evans, D., et al. (1985) A Case-Control Study of BCG and Childhood Tuberculosis in Cali, Colombia. International Journal of Epidemiology, 14, 441-446. https://doi.org/10.1093/ije/14.3.441

[21] Tripathy, S.P. (1987) Fifteen-Year Follow-Up of the Indian BCG Prevention Trial. In: International Union against Tuberculosis, Ed., Proceedings of the 26th IUAT World Conference on Tuberculosis and Respiratory Diseases, Professional Postgraduate Services International, Singapore, 69-72. 
[22] Afanetti, M. and Tissières, P. (2011) Immunité innée du nouveau-né: Spécificités physiologiques et conséquences cliniques. Innate immunity: Physiological aspects and clinical consequences specific to neonatal immunity. Enseignement Supérieur en réanimation pédiatrique. Réanimation, 20, S466-S470. https://doi.org/10.1007/s13546-010-0018-4

[23] Centers for Disease Control and Prevention (1996) The Role of BCG Vaccine in the Prevention and Control of Tuberculosis in the United States. A Joint Statement by the Advisory Council for the Elimination of Tuberculosis and the Advisory Committee on Immunization Practices. MMWR Recommendations and Reports, 45, 1 18.

[24] AlOtaibi, S.M., Almoshadq, A.F., Alghanmi, H.A., Aljuiad, F., Soliman, Y.R., Alzuair, A.T., Alzahrani, O.A., Algarni, A.A. and Ali, M.Y. (2016) Poor Knowledge of Tuberculosis among the Population of Jeddah and Makkah Saudi Arabia, a HighRisk Area. Journal of Tuberculosis Research, 4, 81-91. https://doi.org/10.4236/jtr.2016.43010

[25] Vernaz, A., Gaudart, J., Sallah, K., Casanova, L., Debroise, A., Laporte, R. and Minodier, P. (2014) BCG Vaccination: Survey among Children Less than 5 Years of Age in an Emergency Department. Archives de Pédiatrie, 21, 454-460.

[26] Bouhamam, N., Laporte, R., Boutin, A., Uters, M., Bremond, V., Noel, G., Rodier, P. and Minodier, P. (2012) Relationship between Precariousness, Social Coverage, and Vaccine Coverage: Survey among Children Consulting in Pediatric Emergency Departments in France. Archives de Pédiatrie, 19, 242-247.

[27] Vonasek, B.J., Bajunirwe, F., Jacobson, L.E., Twesigye, L., Dahm, J., Grant, M.J., Sethi, A.K. and Conway, J.H. (2016) Do Maternal Knowledge and Attitudes towards Childhood Immunizations in Rural Uganda Correlate with Complete Childhood Vaccination? PLOS ONE, 11, e0150131. https://doi.org/10.1371/journal.pone.0150131

[28] Odusanya, O.O., Alufohai, E.F., Meurice, F.P. and Ahonkhai, V.I. (2008) Determinants of Vaccination Coverage in Rural Nigeria. BMC Public Health, 8, 381. https://doi.org/10.1186/1471-2458-8-381

[29] Goodman, O.O., Aderibigbe, S.A., Sekoni, O.O., Osagbemi, G.K. and Akande, T.M. (2013) Health Workers Sensitization: Effects on Perceived Quality of Immunization Services among Mothers of under Five Children in Ilorin, North Central Nigeria. Journal of Preventive Medicine and Hygiene, 54, 146-152.

[30] Miyahara, R., Jasseh, M., Gomez, P., Shimakawa, Y., Greenwood, B., Keita, K., Ceesay, S., D’Alessandro, U., Roca, A. (2016) Barriers to Timely Administration of Birth Dose Vaccines in the Gambia, West Africa. Vaccine, 34, 3335-3341. https://doi.org/10.1016/j.vaccine.2016.05.017

[31] Schoeps, A., Ouédraogo, N., Kagoné, M., Sié, A., Müller, O. and Becher, H. (2013) Socio-Demographic Determinants of Timely Adherence to BCG, Penta3, Measles, and Complete Vaccination Schedule in Burkina Faso. Vaccine, 32, 96-102. https://doi.org/10.1016/j.vaccine.2013.10.063

[32] Swana, E.K., Makan, G.Y., Mukeng, C.K., Mupumba, H.I., Kalaba, G.M., Luboya, O.N. and Bangs, M.J. (2016) Feasibility and Implementation of Community-Based Malaria Case Management with Integrated Vector Control in the Democratic Republic of Congo. Malaria Journal, 15, 413. 
Submit or recommend next manuscript to SCIRP and we will provide best service for you:

Accepting pre-submission inquiries through Email, Facebook, LinkedIn, Twitter, etc. A wide selection of journals (inclusive of 9 subjects, more than 200 journals)

Providing 24-hour high-quality service

User-friendly online submission system

Fair and swift peer-review system

Efficient typesetting and proofreading procedure

Display of the result of downloads and visits, as well as the number of cited articles Maximum dissemination of your research work

Submit your manuscript at: http://papersubmission.scirp.org/

Or contact 迆@scirp.org 